\title{
Natural Vibrations of Stepped Arches
}

\author{
Jaan Lellep, Alexander Liyvapuu
}

\begin{abstract}
Free vibrations of elastic arches simply supported at both ends are studied. The arches under consideration have piece wise constant thickness and constant width.
\end{abstract}

Keywords - vibration, arch, crack, elasticity

\section{Introduction}

Plates, shells, beams and arches are the structural elements which have a large variety of applications in the civil engineering and mechanical engineering. Thus the vibration analysis of homogeneous and composite structural elements has not only theoretical but also practical importance.

The foundations of vibrations of beams, plates and shells are presented in the books by Qatu [8], Reddy [9], Vinson and Sierakowski [12].

The book by Soedel [10] contains the governing equations of the dynamic analysis of beams, plates, shells and of certain non-shell structures. The main principles of the analysis of composite and laminated structures can be found in the books by Tuttle [11], Qatu [8], Daniel and Ishai [2], Jones [5], Herakovich [4] and others. Arches are considered by $\mathrm{Xu}$ et al.[14]. In the previous paper by Lellep and Liyvapuu $[6,7]$ a method of determination of natural frequencies of elastic laminated arches was developed.

In the present paper these results are extended to the case of composite and laminated arches of variable thickness.

\section{Problem Formulation and Basic Equations}

Let us consider an elastic circular arch of radius $R$. The arch is simply supported or clamped at the edges at $\varphi=0$ and $\varphi=\beta$ (Fig.1). Here $\varphi$ is the current angle defining the position of the cross-section of the arch. It is assumed that the arch has rectangular cross section with width $b=$ const and the height $h=h_{j}$ for $\varphi \in\left(\alpha_{j}, \alpha_{j+1}\right)$. And $j=0, \ldots, n$. The quantities $\alpha_{j}(j=0, \ldots, n)$ are assumed to be given constants.

It is assumed that due to the initial excitation the arch is performing free vibrations around its equilibrium position. The goal of the paper is to determine the natural frequencies of the free vibrations. In order to solve this task we shall solve the governing equations appropriate to the posed problem.

It is assumed that the arch under consideration is made of a composite or laminated material.

Jaan Lellep

Institute of Mathematics / University of Tartu

Estonia

\section{Alexander Liyvapuu}

Institute of Mathematics / University of Tartu

Estonia

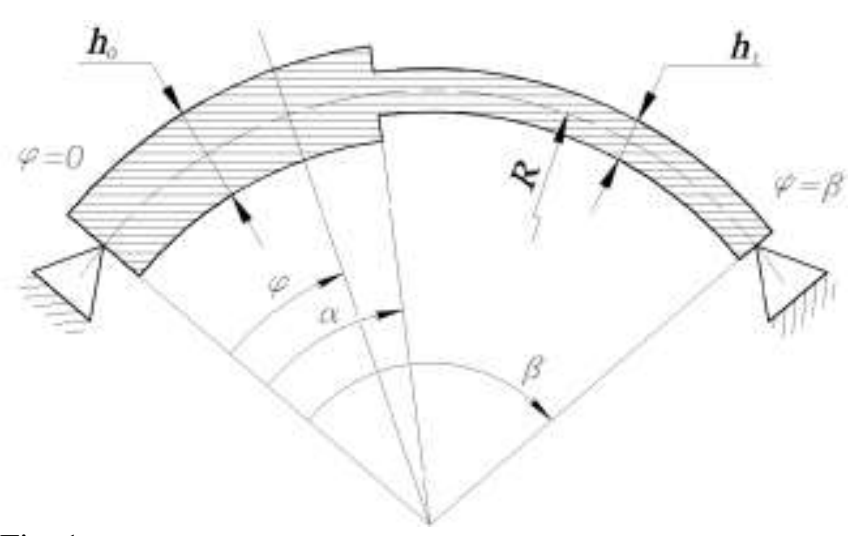

Fig. 1

The constitutive equations of layered composite arch can be presented (see Reddy [9], Vinson and Sierakowski [12]) with the help of the membrane force and bending moment

$$
\begin{gathered}
N=\sum_{k=1}^{K_{j}} \int_{z_{k}}^{K_{j}} \sigma_{s} d z \\
M=\sum_{k=1}^{z_{k+1}} \int_{z_{k}}^{z_{k}} \sigma_{s} z d z
\end{gathered}
$$

here $z$ stands for the local coordinate directed through the thickness of the arch and $K_{j}$ is the number of layers, $z_{k+1}-z_{k}=h_{k j}$ being the thickness of a layer number $k$ in the interval $\left(\alpha_{j}, \alpha_{j+1}\right)$. In (1) $\sigma_{s}$ is the normal stress acting on the face $\varphi=$ const of an increment element of the arch It can be seen from (1) that the both, the bending moment and the membrane force are engendered by the same normal stress $\sigma_{s}$.

Evidently, tangential stress component $\sigma_{s \varphi}$ is to be taken into account as well. However, this stress component induces the shear force

$$
Q_{s}=\int_{-\frac{h}{2}}^{\frac{h}{2}} \sigma_{s \varphi} d z .
$$

On the other hand, the shear force can be eliminated from the set of the governing equations and thus, it is not considered in the following analysis.

The Hooke's law for a multilayered arch reads (see Reddy [9])

$$
\begin{gathered}
N=A \varepsilon+B \varkappa, \\
M=B \varepsilon+D \varkappa .
\end{gathered}
$$

In (2) $\varepsilon$ and $\mathcal{H}$ stand for the strain and curvature, respectively, expressed as 


$$
\varepsilon=\frac{1}{R}\left(U^{\prime}+W\right), \quad \varkappa=-\frac{1}{R^{2}}\left(-U^{\prime}+W^{\prime \prime}\right)
$$

where $U$ and $W$ stand for the displacements in the tangential and transverse direction, respectively.

It is reasonable to assume that there is no extension of the axis of the arch. This means that $\varepsilon=0$ and according to the first relation in (3) $U^{\prime}=-W$.

Therefore in this case

$$
\varkappa=-\frac{1}{R^{2}}\left(W+W^{\prime \prime}\right)
$$

and

$$
M=-\frac{D_{j}}{R^{2}}\left(W+W^{\prime \prime}\right)
$$

for $\in\left(\alpha_{j}, \alpha_{j+1}\right)$.

In the case of unidirectionally reinforced laminae with elastic coefficients $E_{1}^{(k)}, v_{12}^{(k)}$ and $E_{2}^{(k)}, v_{21}^{(k)}$ in two perpendicular directions one has

$$
D_{j}=\frac{1}{3} \sum_{k=1}^{K_{j}} Q_{11}^{(k)}\left(h_{k j}^{3}-h_{(k-1) j}^{3}\right)
$$

where

$$
Q_{11}^{(k)}=\frac{E_{1}^{(k)}}{1-v_{12}^{(k)} v_{21}^{(k)}} .
$$

Note that the two perpendicular directions in the material necessary for the validity of (6),(7) are to be chosen so that one of these coincides with the alignment of fibers in the matrix.

The equilibrium equations of an element of the arch can be put into the form (see Soedel [10], Lellep, Liyvapuu [6, 7])

$$
M^{\prime \prime}+M-\rho_{j} h_{j} \ddot{W}=0
$$

Where $\rho_{j}$ is the mass per unit length of the arch. Here dots denote the differentiation with respect to time $t(\ddot{W}$ is the acceleration of an element of the arch).

As the free vibrations of an arch simply supported at the both ends will be treated the boundary conditions are at $\varphi=0$

and

$$
W(0, t)=0, \quad M(0, t)=0
$$

$$
W(\beta, t)=0, \quad M(\beta, t)=0 .
$$

\section{Solutions of the Equation of Vibration}

The equation of motion (8) is a linear fourth order equation with constant coefficients. Thus one can employ the method of separation of variables. For this purpose one can present the deflection as

$$
W(\varphi, t)=W(\varphi) \cdot T(t)
$$

where $w(\varphi)$ depends only on the current angle and $T(t)$ depends only on the time. Differentiating $W$ with respect to the coordinate one easily obtains

$$
W^{\prime}(\varphi, t)=w^{\prime}(\varphi) \cdot T(t)
$$

Calculating the second derivative results in

$$
W^{\prime \prime}(\varphi, t)=w^{\prime \prime}(\varphi) \cdot T(t)
$$

Evidently,

$$
W^{\prime \prime \prime}(\varphi, t)=w^{\prime \prime \prime}(\varphi) \cdot T(t)
$$

and

$$
W^{I V}(\varphi, t)=w^{I V} \cdot T(t)
$$

Similarly, one can determine the acceleration.

$$
\ddot{\mathrm{W}}(\varphi, t)=w(\varphi) \cdot \ddot{T} \text {. }
$$

Substituting the derivatives (12) in (9) and separating variables leads to the equations

$$
\ddot{T}+\ddot{\omega}^{2} T=0
$$

and

where

$$
w^{I V}+2 w^{\prime \prime}+w\left(1-k_{j}^{2}\right)=0
$$

$$
k_{j}=\sqrt{\frac{12 \rho R^{4}\left(1-v^{2}\right) \omega^{2}}{E h_{j}^{2}}}
$$

In (13) and (15) $\omega$ stands for the frequency of natural vibrations.

It immediately yields from (13) that

$$
T(t)=A_{1} \sin \omega t+A_{2} \cos \omega t
$$

Assuming that the initial conditions are presented as

one can determine

$$
W(\varphi, 0)=0, \quad \dot{W}\left(\varphi_{*}, 0\right)
$$

and

$$
A_{2}=0
$$

$$
A_{1}=\frac{v_{*}}{\omega w\left(\varphi_{*}\right)} .
$$

Thus one can state that

$$
T(t)=A_{1} \sin \omega t
$$

In order to obtain the general solution of the equation (14) one has to compile the characteristic equation

$$
\lambda_{j}^{4}+2 \lambda_{j}^{2}+1-k_{j}^{2}=0 .
$$

It immediately follows from (18) that

$$
\lambda_{j}^{2}=-1 \pm k_{j}
$$

and

$$
\lambda_{j}= \pm \sqrt{-1 \pm k_{j}}
$$

The subsequent analysis shows that $-1+k_{j}<0$ and $1+k_{j}>0$ for each $j=0, \ldots, n$.

Introducing the notation

$$
\mu_{j}^{2}=1-k_{j}, \quad v_{j}^{2}=1+k_{j}
$$

one can present the general solution of the equation (14) as

$$
\begin{aligned}
w=C_{j 1} \cosh \mu_{j} \varphi & +C_{j 2} \sinh \mu_{j} \varphi \\
& +C_{j 3} \cos v_{j} \varphi+C_{j 4} \sin v_{j} \varphi
\end{aligned}
$$

where $C_{j 1}, C_{j 2}, C_{j 3}$ and $C_{j 4}$ are arbitrary constants and in according to (22) 
and

$$
\mu_{j}=\sqrt{1-\omega R^{2} \sqrt{\frac{\bar{\rho}_{j}}{D_{i}}}}
$$

$$
v_{j}=\sqrt{1+\omega R^{2} \sqrt{\frac{\bar{\rho}_{j}}{D_{i}}}}
$$

for each $j=0, \ldots, n$.

The arbitrary constants will be determined with the help of boundary and intermediate conditions. The latters are suggested by Chondros, Dimarogonas, Yao[1], Dimarogonas [3] in order to model the influence of a crack on the natural frequency of beam.

\section{Boundary Conditions and Intermediate Requirements}

In the case of the arch simply supported at both ends the bending moment and the transverse deflection equal to zero at both ends.

This means, that the conditions (10) hold good. Making use of (11) and taking into account that $T(t) \not \equiv 0$ one can conclude that

and

$$
w(0)=0, \quad w^{\prime \prime}(0)=0
$$

$$
w(\beta)=0, \quad w^{\prime \prime}(\beta)=0 .
$$

Due to physical considerations the deflection $W$ (and thus $w(\varphi))$, also bending moment $M$ and the shear force $Q=\frac{1}{R} M^{\prime}$ must be continuous at $\varphi=\alpha_{j}(j=1, \ldots, n)$.

Bearing in mind (11) one can write

$$
\begin{gathered}
w\left(\alpha_{j}-0\right)=w\left(\alpha_{j}+0\right), \\
w^{\prime}\left(\alpha_{j}-0\right)=w^{\prime}\left(\alpha_{j}+0\right), \\
D_{j-1}\left(w\left(\alpha_{j}-0\right)+w^{\prime \prime}\left(\alpha_{j}-0\right)\right)= \\
=D_{j}\left(w\left(\alpha_{j}+0\right)+w^{\prime \prime}\left(\alpha_{j}+0\right)\right), \\
D_{j-1}\left(w^{\prime}\left(\alpha_{j}-0\right)+w^{\prime \prime \prime}\left(\alpha_{j}-0\right)\right)= \\
=D_{j}\left(w^{\prime}\left(\alpha_{j}-0\right)+w^{\prime \prime \prime}\left(\alpha_{j}+0\right)\right)
\end{gathered}
$$

for each $j=1, \ldots, n$ where

$$
D_{j}=\frac{E h_{j}^{3} b}{12\left(1-v^{2}\right) R^{2}} .
$$

\section{v. Natural Frequencies of the Weakened Arch; Numerical Results}

In order to determine the eigenfrequencies of the arch with cracks one has to determine the solution (23) satisfying all boundary and intermediate conditions. The set of intermediate conditions is presented in the form (25), (26). Thus the system of boundary conditions (24) with intermediate conditions (25) consists of $4 n+4$ algebraic equations with the determinant

$$
\Delta=\left|\begin{array}{cccc}
\xi_{1} & \xi_{2} & \xi_{3} & \xi_{4} \\
\mu_{0} \xi_{5} & v_{0} \xi_{6} & \mu_{1} \xi_{7} & -v_{1} \xi_{8} \\
\mu_{0}^{2} \xi_{1} & -v_{0}^{2} \xi_{2} & \mu_{1}^{2} \xi_{3} & -v_{1}^{2} \xi_{4} \\
\mu_{0}^{3} \xi_{5} & -v_{0}^{3} \xi_{6} & \mu_{1}^{3} \xi_{7} & v_{1}^{3} \xi_{8}
\end{array}\right|=0 .
$$

There are substitutions in the determinant

$$
\begin{gathered}
\xi_{1}=\sinh \mu_{0} \alpha \\
\xi_{2}=\sin v_{0} \alpha \\
\xi_{3}=\tanh \mu_{1} \beta \cdot \cosh \mu_{1} \alpha-\sinh \mu_{1} \alpha \\
\xi_{4}=\tan v_{1} \beta \cdot \cos \nu_{1} \alpha-\sin \nu_{1} \alpha \\
\xi_{5}=\cosh \mu_{0} \alpha \\
\xi_{6}=\cos v_{0} \alpha \\
\xi_{7}=\tan v_{1} \beta \cdot \sin v_{1} \alpha+\cos \nu_{1} \alpha \\
\xi_{8}=\tanh \mu_{1} \beta \cdot \sinh \mu_{1} \alpha-\cosh \mu_{1} \alpha
\end{gathered}
$$

As the linear system is a homogeneous system the determinant $\Delta$ must vanish.

The equation $\Delta=0$ is solved numerically using the computer code Matlab. The results of calculations are presented in Fig.2 -Fig.5.

The curves presented in Fig. 2 correspond to the arch with $R=1 \mathrm{~m}, h_{0}=0.01 \mathrm{~m}, h_{1}=0.02 \mathrm{~m}$. The material is steel with $E=2.1 \cdot 10^{11} \mathrm{~Pa}, v=0.3, \rho=7865 \mathrm{~kg} / \mathrm{m}^{3}$.

The dependence of the natural frequency on the radius of the arch is shown in Fig.2. Different curves in Fig.2 correspond to the different radii. Here the central angle of the $\operatorname{arch} \beta=1.5$ whereas $h_{0}=0.05 \mathrm{~m}$ and $h_{1}=0.02 \mathrm{~m}$.

It can be seen from Fig. 2 that the greater is the radius of the arch with the lower is its natural frequency. Thus the natural frequency of a straight beam is lower than that of a curved beam fixed at ends at the same manner.

The results presented herein correspond to arches with a single step location at $\varphi=\alpha_{1}$. For the distinctness the step coordinate is denoted by $\alpha=\alpha_{1} / \beta$.

In Fig. 3 the natural frequency $\omega$ versus $\alpha$ is depicted for different values of the central angle $\beta$ of the arch. It can be seen from Fig. 3 that when the step location moves towards the right hand end of the arch the natural frequency monotonically increases, as might be expected.

Evidently, the points of curves where $\alpha=0$ correspond to natural frequencies of arches with constant thickness $h=$ $h_{0}$. Similarity the right hand ends of curves for $\alpha=1$ correspond to arches with constant thickness $h=h_{1}$.

The natural frequency $\omega$ versus the quantity $\alpha$ is presented in Fig. 4 for different ratios of the thicknesses

$$
\gamma=\frac{h_{1}}{h_{0}} \text {. }
$$


The curves presented in Fig. 4 correspond to the cases $h_{1}=0.3 h_{0}, h_{1}=0.4 h_{0}, h_{1}=0.5 h_{0}$,

$h_{1}=0.6 h_{0}, \quad h_{1}=0.7 h_{0}$. Here $\beta=1.5 \mathrm{rad}$.

It can be seen from Fig. 4 that when the step $\alpha$ moves towards the right hand end of the arch the natural frequency decreases.

In Fig.5 similar curves are presented for various materials including bronze, concrete, glass and aluminum. The lowest values of $\omega$ correspond to the arch made of concrete.

The highest natural frequencies are revealed by the vibration of the arch made of glass.

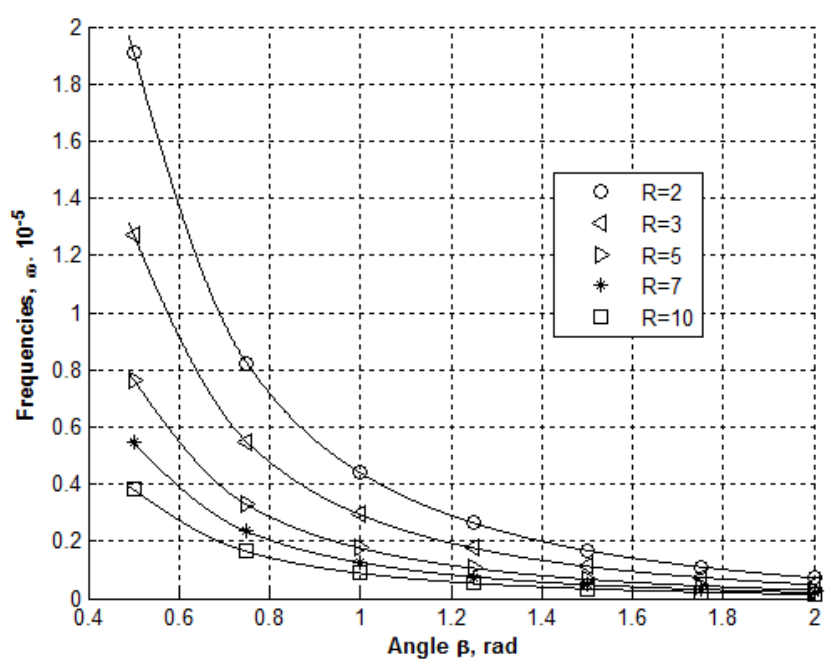

Fig. 2. Dependence of the natural frequency on the angle of the arch

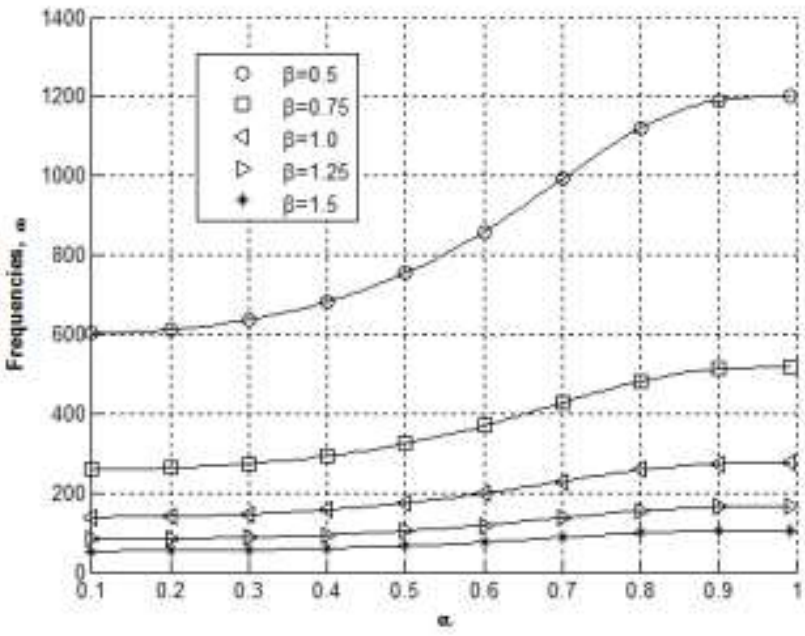

Fig. 3. Dependence of the natural frequency of the arch on the step location

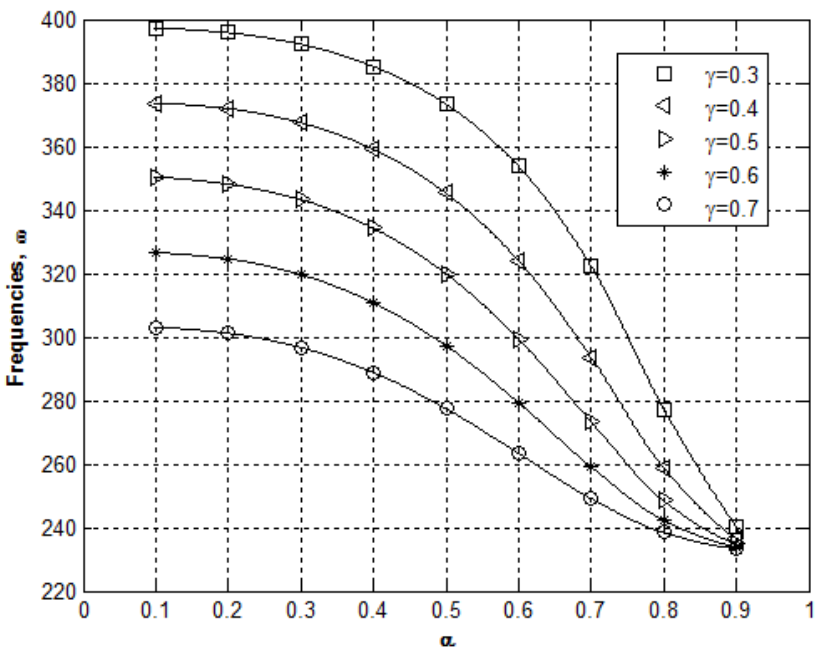

Fig. 4. Dependence on the ratio of thicknesses

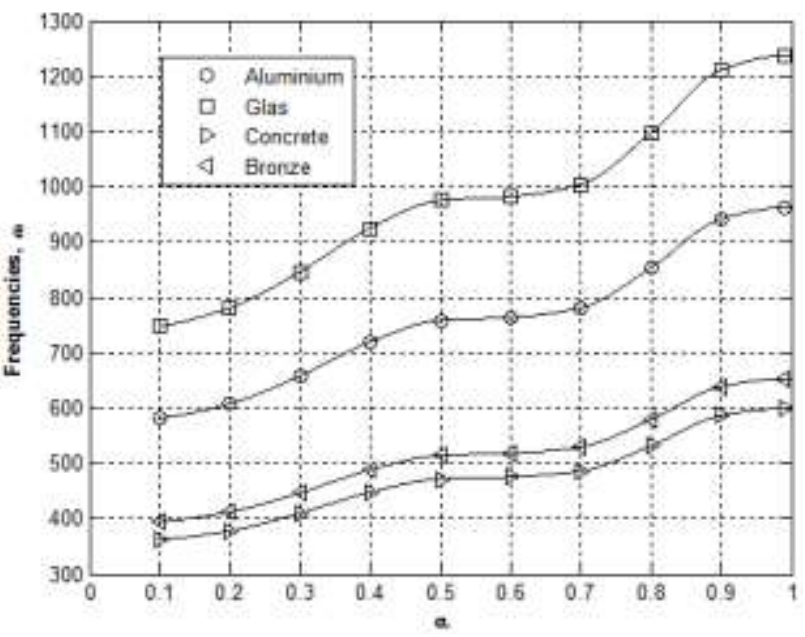

Fig. 5. Dependence on material of the arch made

\section{vI. Concluding Remarks}

A vibration analysis is undertaken for elastic circular arches. The theory of vibration is developed for arches made of unidirectionally reinforced composites or laminated material. The arches have piece wise constant thicknesses and constant width. An exact method has been developed for determination of eigenfrequencies of stepped arches.

Numerical calculations revealed the matter that the natural frequency of one-stepped arches rapidly increases if the step moves towards the thinner edge of the arch. 


\section{Acknowledgment}

The partial support from the Estonian Science Foundation grant ETF 9110 "Optimization of structural elements" and from the Institutional research funding IUT 20-57 of the Estonian Ministry of Education and Research are gratefully acknowledged.

\section{References}

[1] T.J. Chondros, A.D. Dimarogonas, J. Yao. "A continuous cracked beam vibration theory.” J. Sound Vibration, 1998, 215(1), pp.17-34.

[2] I.M. Daniel, O. Ishai,"Engineering Mechanics of Composite Materials." Oxford Univ. Press, 2006.

[3] A.D. Dimarogonas, "Vibrations of cracked structures: a state of the art review," Eng. Fracture Mech., 1996, 55, pp. 831-857.

[4] C.T. Herakovich,"Mechanics of Fibrous Composites." Wiley, NewYork, 1998.

[5] R.M. Jones,"Mechanics of Composite Materials." Taylor and Francis, Philadelphia, 1999.

[6] J. Lellep, A. Liyvapuu,"Natural vibrations of elastic arches with cracks." In: J.Lellep, E.Puman (Ed-s), Optimization and Analysis of Structures, Tartu Univ. Press, Tartu, 2013, pp. 64-69.

[7] J. Lellep, A. Liyvapuu,"Free vibrations of elastic laminated arches." In: J.Lellep, E.Puman (Ed-s), Optimization and Analysis of Structures, Tartu Univ. Press, Tartu, 2015, pp. 52-58.

[8] M.S. Qatu,"Vibrations of Laminated Plates and Shells." Elsevier, New-York, 2004.

[9] J.N.Reddy, "Mechanics of Laminated Composite Plates and Shells. Theory and Analysis." CRC Press, 2004.

[10] W.Soedel, "Vibrations of Shells and Plates." Marcel Dekker, NewYork, 2004.

[11] M.E.Tuttle, "Structural Analysis of Polymeric Composite Materials." Marcel Dekker Press, New-York, Basel, 2004.

[12] J. Vinson, R.L. Sierakowski, "The Behavior of Structures Composed of Composite Materials," Kluwer, 2002.

[13] Y.Xiang, C.M.Wang, "Exact buckling and vibration solutions for stepped rectangular plates," Journal of Sound and Vibration, 2002, 250, pp. 503-217.

[14] Y.Xu, X.Gu, B.Zhao, R.Zhou,"In-plane elastic stability of arches under a radial concentrated load." Engineering, 2014, 6, pp.572583. 